\title{
"Dovetail" gastric resection: a novel model in the treatment of gastrointestinal stromal tumors
}

\author{
Denise Gambardella ${ }^{1}$ Angelo Aldo Schicchi ${ }^{2}$ Andrea Boccuto ${ }^{2}$, Vito Bilotta ${ }^{2}$ Ettore Caruso ${ }^{3}$, Luigino \\ Borrello ${ }^{4}$, Manfredo Tedesco ${ }^{4}$
}

'Department of Medical and Surgical Sciences, University of Catanzaro, Catanzaro, Italy.
2Operative Unit of Endoscopy, Director Angelo Aldo Schicchi, "Giovanni Paolo II" Hospital, Lamezia Terme, Italy.
'Division of Endocrine and Minimally Invasive Surgery, University of Messina, Italy.
${ }^{4}$ Department of General Surgery, Director Manfredo Tedesco, "Giovanni Paolo II" Hospital, Lamezia Terme, Italy.

\section{To Cite}

Gambardella D, Schicchi A A, Boccuto A, Bilotta V, Caruso E, Borrello L, Tedesco M.

"Dovetail" gastric resection: a novel model in the treatment of gastrointestinal stromal tumors. J Gastric Surg 2020; 2(4)

\section{Publication history}

Received: October 20, 2020

Accepted: October 30, 2020

Article in press: November 04, 2020

Published online: November 11, 2020

\section{*Correspondence to}

Denise Gambardella, MD

Department of General Surgery, University of Catanzaro, Catanzaro, Italy. gambardelladenise@gmail.com

\begin{abstract}
Gastrointestinal stromal tumors (GISTs) are the most common malignant subepithelial lesions of the gastrointestinal tract. The prognosis of this disease is associated with the tumor size and mitotic index. The standard treatment of a GIST without metastasis is surgical resection. The stomach is the most frequent site of the disease and many technical solutions have recently been proposed as shown also by the dramatic increase in publications on this field. There are many studies that suggest laparoscopy may be an acceptable surgical treatment option compared to open surgery for gastric GIST. However, open surgery assumes great importance in large tumors located in difficultto-access locations. Here, we present a case involving a 60 -year-old man who was diagnosed with gastric antrum GIST, according to a preoperative examination and postoperative pathology. Then, the patient successfully underwent an atypical gastric resection. We proposed a novel surgical technique to be considered in case of gastric benign disease or GIST localized at the gastric antrum.
\end{abstract}

Keywords:

Gastrointestinal stromal tumors, GIST, gastric resection. 


\section{Background}

Gastrointestinal stromal tumors (GISTs) are the most frequent malignant subepithelial lesions of the digestive tract. Their origin is from the interstitial cells of Cajal located within the muscle layer and their main characteristic is an overexpression of the receptor tyrosine kinase KIT. The prognosis is associated with tumor size and mitotic index while standard treatment is surgical resection for non-advanced stages of disease. The surgeon's objective is to achieve the R0 resection with the largest possible free margin.[1] Laparoscopic surgery is a valid surgical treatment option compared to open surgery for gastric GIST.[2] It is possible to choose between an open surgery or a laparoscopic resection for gastric GIST in selected patients, especially for tumors less than $5 \mathrm{~cm}$, or located on the anterior wall, greater curvature, less curvature. In these cases, laparoscopic surgery demonstrated better short-term outcomes than open resection.[3]

Large lesions located at the level of the anthro-pyloric region often need major resections. In this technical note, we propose a new gastric resection for giant GISTs of the gastric antrum as an alternative to Billroth I (B-I) and Billroth II (B-II) and Roux-en-Y (R-Y) reconstructions burdened by perioperative and postoperative effects.

\section{Case Report}

A 60-year-old male patient with a history of hypertension was admitted with the chief complaint of upper abdominal pain for 3 month; The pain abdominal distension, and discomfort that was accompanied by a sour regurgitation and weight loss. He had no family history of malignant tumors. Routine blood, routine urine, blood biochemical tests were normal, and the tumor marker test results were all within normal ranges. Gastroscopy revealed a giant smooth bulge covered with normal mucosa with a maximum diameter of $7 \mathrm{~cm}$ in the gastric antrum that indicated a probable stromal tumor (Figure 1).

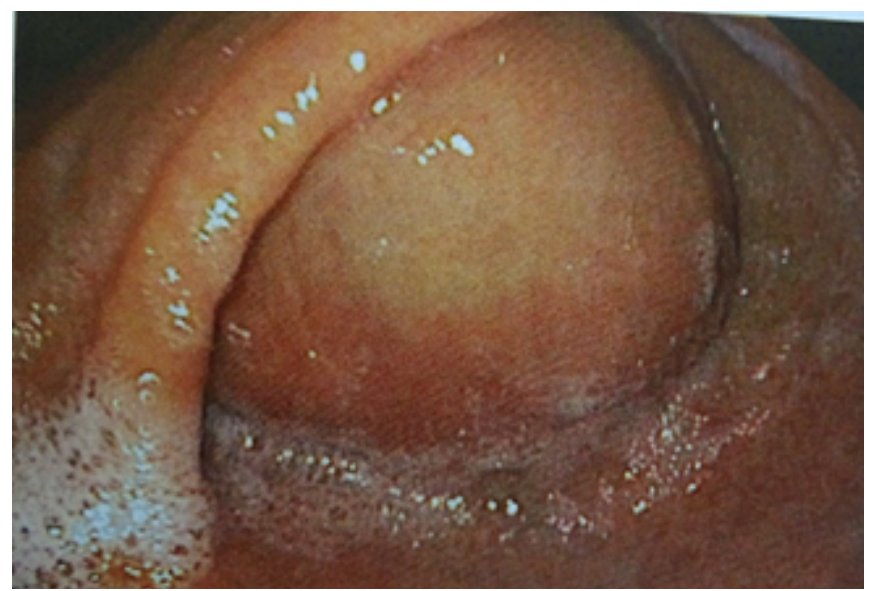

Figure 1: Gastroscopy revealed a giant smooth bulge covered with normal mucosa with a maximum diameter of $7 \mathrm{~cm}$ in the gastric antrum that indicated a probable stromal tumor.

Computed tomography confirmed the presence of this lesion in the gastric antrum, without adenopathy or liver metastasis. After multidisciplinary meeting, we suspected the diagnosis of stromal tumour and we decided to perform open surgery. The patient underwent laparotomy and we performed an atypical gastric resection (Figure 2-3).

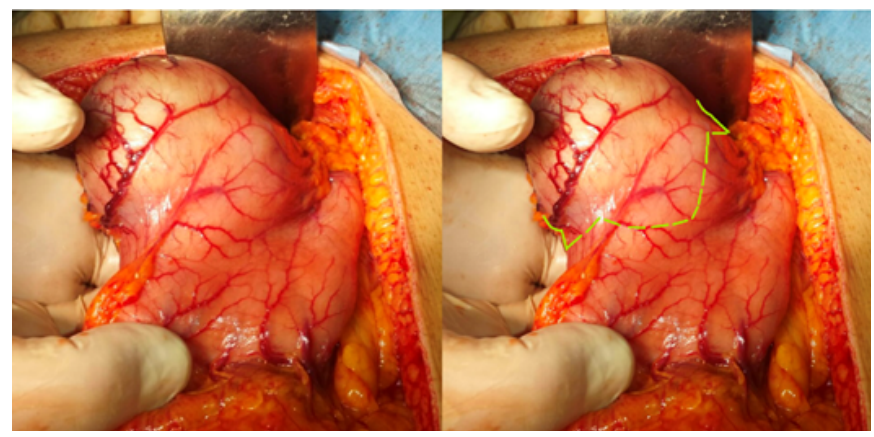

Figure 2: Neoplastic region and surgical resection.

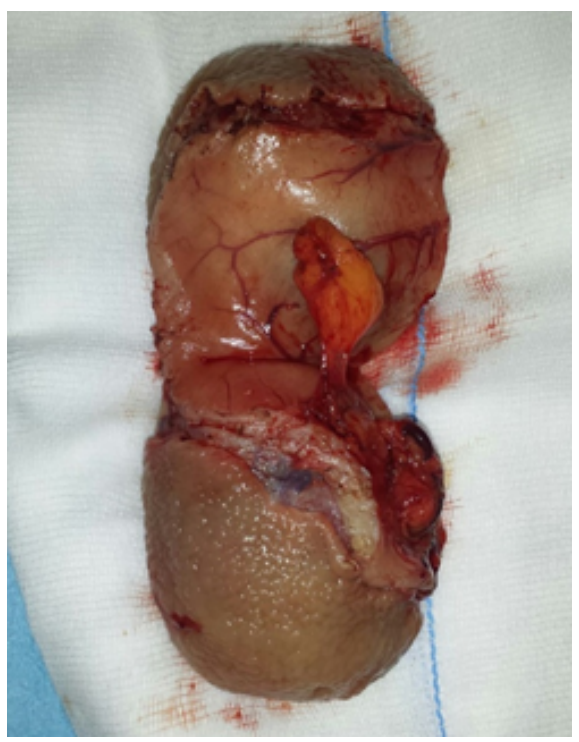

Figure 3: Surgical specimen.

The post-operative course was uneventful. The patient recovered well and was discharged six days after his operation. The histopathology of the gastric fundus indicated GIST; features included spindleshaped cells, no significant atypia, signs of extensive necrosis, mitotic $5 / 50 \mathrm{HPF}$. Immunohistochemistry demonstrated the following: CD117 (+), CD34 (+), SMA (+), S-100 (-), and vimentin $(+)$ ( Figure 4$)$.

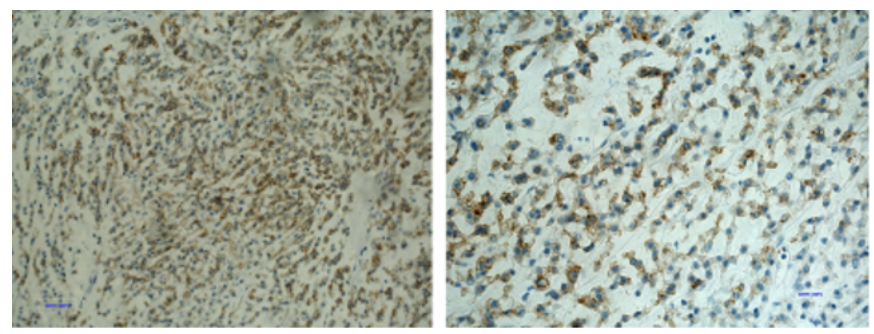

Figure 4: Cytoplasmic and membrane expression of CD117.

All margins were negative. The fragment of oment and the lymph nodes were unlawful. Combining these immunohistochemical results with the preoperative examination, intraoperative findings, and postoperative pathology, a diagnosis of gastric antrum GIST was made. The patient's prognosis, according with the tumor size and mitotic index, was an intermediate risk. Considering the prognosis, oral imatinib was administrated. The patient regularly underwent reexaminations with 
gastroscopy and abdominal CT scan. There were no recurrences or distal metastases detected after 24 months of follow up.

\section{Technical Note}

A small incision was made between the xiphoid and umbilicus. First, an exploratory laparotomy was performed.

The greater omentum of the stomach was dissected from the middle portion of the transverse colon, exposing the posterior wall of the stomach and opening the lesser sac. The gastroepiloic artery and vein at the greater curve are ligated and transected, preserving the arcade vessels of the proximal part of the stomach. The access to the epiploon retrocavity allowed to control the integrity of the gastric wall. The omentum minus was opened along the lesser gastric curvature. The right gastric artery was exposed and its branches tied. Gastrotomy was performed on the antral side. The eversion of the anterior gastric wall revealed a giant smooth bulge covered with normal mucosa with a maximum diameter of $7 \mathrm{~cm}$ in the gastric antrum that indicated a possible stromal tumor. "Dovetail" gastric wall resection including the tumor was performed while maintaining a safety margin of at least $2 \mathrm{~cm}$. This semi-circular resection of the anterior and posterior gastric wall, posteriorly partial, allowed the removal of the lesion. Lymphnodes stations \#5 and \#6 were also included. A termino-terminal anastomosis was performed between the wall of the gastric body and the proximal portion of the pylorus with manual suturing. A nasogastric tube was placed and then removed on the fourth post-operative day.

\section{Discussion}

The most effective treatment for gatric GIST is radical resection. This is still the only treatment option that provides the highest chance of medium to long-term overall and disease-free survival. Gastrointestinal reconstruction procedure options after distal or subtotal gastrectomy for patients with gastric disease are still controversial.[4]

The three possible reconstructions for patients with gastric cancer or benign gastric disease are reconstructions B-I and B-II, R-Y. However, patients undergoing B-I and B-II reconstruction often show reflux symptoms as a complication. On the contrary, the R-Y reconstruction is superior to the traditional B-I and B-II reconstructions in the prevention of reflux symptoms and in preventing gastritis that seems to increase the risk of gastric carcinogenesis. However, the R-Y is a more complex reconstruction and some patients show the delayed gastric emptying syndrome, known as Roux stasis syndrome. To avoid complications, some technical shrewdnesses are necessary as an adequate length of the alimentary limb.[5]

We proposed a novel atypical gastric resection technique for gastric antral GIST as an alternative to B-I, B-II and $\mathrm{R}-\mathrm{Y}$ reconstructions that, as previously reported, are burdened by negative postoperative effects. The ideal reconstruction method should fit the patient's physical condition, reduce the risk of postoperative complications, and improve the patient's quality of life. The reconstruction method is often a matter of the surgeon's preference. We choose the reconstruction method on the basis of the tumor location. For this Gastric antrum GIST we prefer to do a semi-cicular "Dovetail" resection including the tumor, maintaining a safety margin of $2 \mathrm{~cm}$. This resection strategy led to good functional results, such as increased food intake, post-operative body weight retention and no complaints of postprandial symptoms. The "Dovetail" method has the physiological advantage of allowing the food to pass through the duodenum, with a better effect on digestion and absorption of nutrients. The natural transition improves long-term nutritional status and quality of life. We suggest to perform this type of atypical resection for GISTs or benign gastric tumors involving the antrum that seems to be effective and safe with optimal clinical and oncological outcomes.

\section{Acknowledgements}

None

\section{Contributors}

DG, AAS, AB, VB, EC, LB, MT conceptualized and designed the study, acquired, and analyzed data, interpreted the study results, drafted the manuscript, and critically revised the final version of the manuscript.

\section{Funding}

No funding was received for this study.

\section{Competing interests}

The authors have no conflict of interest to disclose, and no other funding or financial relationship with the surgical industry. This report does not endorse any specific Company, set of endoscopic or monitoring equipment.

\section{Availability of data and materials}

Further information is available from the corresponding author on reasonable request.

\section{Ethics approval \\ Not applicable.}

\section{Provenance and peer review}

Not commissioned; externally peer reviewed.

\section{Open access}

This is an Open Access article distributed in accordance with the Creative Commons Attribution NonCommercial (CC BY-NC 4.0) license, which permits others to distribute, remix, adapt, build upon this work noncommercially, and license their derivative works on different terms, provided

the original work is properly cited and the use is non-commercial. See: http://creativecommons.org/ licenses/by-nc/4.0/

\section{References}

[1] Akahoshi K, Oya M, Koga T, Shiratsuchi Y. Current clinical management of gastrointestinal stromal tumor. World J Gastroenterol. 2018;24:2806-17.

[2] Ohtani H, Maeda K, Noda E, Nagahara H, Shibutani M, Ohira $\mathrm{M}$, et al. Meta-analysis of laparoscopic and open surgery for gastric gastrointestinal stromal tumor. Anticancer Res. 2013;33:5031-41.

[3] Wang Y, Li Z, Shan F, Miao R, Xue K, Li Z, et al. [Current status 
of diagnosis and treatment of early gastric cancer in China--Data from China Gastrointestinal Cancer Surgery Union]. Zhonghua Wei Chang Wai Ke Za Zhi. 2018;21:168-74.

[4] Cai Z, Zhou Y, Wang C, Yin Y, Yin Y, Shen C, et al. Optimal reconstruction methods after distal gastrectomy for gastric cancer: A systematic review and network meta-analysis. Medicine (Baltimore). 2018;97:e10823.

[5] Wu JZ, Fukunaga T, Oka S, Kanda S, Ishibashi Y, Yube Y, et al Comparative study of outcomes of Roux-en-Y reconstruction and Billroth reconstruction performed after radical distal gastrectomy. Asian J Surg. 2019;42:379-85. 